\title{
Re-thinking microbiology/infection control education to enhance the practice-readiness of health professional students: More than just a curriculum issue
}

\author{
Jennifer L. Cox \\ Charles Sturt University, Australia \\ jcox@csu.edu.au \\ Maree Donna Simpson \\ Charles Sturt University, Australia \\ masimpson@csu.edu.au \\ Will Letts \\ Charles Sturt University, Canada \\ wletts@csu.edu.au \\ Heather Cavanagh \\ Charles Sturt University, Australia \\ hcavanagh@csu.edu.au
}

\begin{abstract}
Undergraduate education in the health professions is intended to produce competent health professional graduates. Infection prevention and control (IPC) is a necessary element of daily practice in many health professions, to safeguard patients and staff, however previous research has established poor knowledge and implementation of IPC precautions despite escalating rates of potentially lethal healthcare-associated infections (HAIs) across the globe. This paper will discuss three key areas of influence for graduates' IPC knowledge, intentions and practice: perceptions of science, health behaviour beliefs (perceived risk and self-efficacy) and applied knowledge (microbiology). To date, each of these areas have been researched individually however there is an urgent need for improved synthesis and integration of these factors in curriculum planning and design, both inside and outside the classroom, to enhance the development of competent, workready graduates.
\end{abstract}

\section{Keywords}

Nursing education, curriculum, integration, misperception, microbiology, infection prevention and control (IPC), health care workers (HCW) 


\section{Introduction}

In the healthcare professions, as in any profession, it is imperative that students are able to transfer their undergraduate knowledge and skills into practice to be competent practitioners. With the rapid rise of resurgent infections such as Ebola and multi-drug resistant tuberculosis (MDR-TB), the need for healthcare workers (HCWs) to be able to safely work with patients with transmissible diseases (i.e. use the right protection for that agent), has become more important than ever. Indeed the ability of HCWs to appropriately apply good infection prevention and control (IPC) practices may, in fact, be a matter of life and death. Despite this impetus and the seriousness of the consequences, the infection control knowledge and practice of health professional graduates, globally, has been reported to be inadequate and unsatisfactory (Cox, Simpson, Letts, \& Cavanagh, 2014; Liu, Curtis, \& Crookes, 2014), potentially exposing themselves, their colleagues and their patients to life-threatening diseases.

Using nursing education as a case study, this paper will discuss the interaction among behavioural factors, self-efficacy and perceptions of science (including microbiology) and risk that influence students' IPC intentions and practice and the need for these factors to be considered in context to enhance behavioural outcomes.

\section{Infection control - why it matters}

Healthcare-associated infections (HAIs), infections that people contract whilst being cared for in a healthcare facility, continue to pose a significant burden to healthcare systems worldwide. These potentially preventable infections have significant health, social and economic consequences and are recognised as a major risk to patient safety (Collignon, Wilkinson, Gilbert, Grayson, \& Whitby, 2006). In recent years, increasing resistance to antibiotics has seen some bacteria previously of little concern to human health become a significant source of morbidity and mortality (ACSQHC, 2013). A 2009 report (Scott, 2009) estimated the direct medical costs of all HAIs to US hospitals to be between USD28.4 and USD45 billion annually. In Australia, there are approximately 200,000 cases of HAI annually with a resultant loss of an estimated two million bed days (Cruickshank \& Ferguson, 2008). The rapid increase in HAI rates has been attributed to a number of factors including increasing antibiotic resistance, overcrowding of hospitals and poor compliance with infection prevention and control precautions by healthcare workers (Clements et al., 2008; Gammon \& Gould, 2005). Cross-infection of patients by HCWs with contaminated hands remains a significant driver of HAIs (Burke, 2003) and it is widely recognised that appropriately applied infection control precautions, particularly hand hygiene, can effectively reduce the rate of HAIs in healthcare settings (Harbarth, Sax, \& Gastmeier, 2003). Despite this there is a substantial body of evidence to indicate that HCWs are inconsistent in their application of infection control precautions (Gammon \& Gould, 2005) and that hand hygiene practice, in particular, is suboptimal in most healthcare settings (Allegranzi \& Pittet, 2009). 
A variety of factors such as understaffing (Clements et al., 2008), skin irritation by hand-hygiene agents such as antiseptic hand washes (Huggonet \& Pittet, 2000) and misconceptions about microbiological principles (Prieto \& Clark, 2005) have been cited in the literature as barriers to compliance with infection control policies. Compliance is also likely to be influenced by professional attitudes and beliefs, perceived risk of infection, and workplace culture (DeJoy, Gershon, \& Schaffer, 2004; Mutha et al., 1999; Whitby, McLaws, \& Ross, 2006). Despite a growing body of research in the area of infection control practice, evidence of the single most effective strategy to improve hand hygiene compliance is inconclusive (Gould, Drey, Moralejo, Grimshaw, \& Chudleigh, 2008).

\section{Education}

Sherwood (2011) acknowledged the central role of undergraduate education in transforming safety practices in the health care profession:

Education is regarded as the bridge to quality, the link to creating the changes needed in the system. Aims to improve quality and safety demand transformation of health professions education by integrating quality and safety science into curricula.

(Sherwood, 2011, p. 227)

\section{Competency development}

As one of the core competencies of nursing practice, infection prevention and control forms an integral component of undergraduate nursing programs worldwide. The Australian Registered Nurse Competency Standards (Nursing and Midwifery Board of Australia, 2006) defined competence as "the combination of skills, knowledge, attitudes, values and abilities that underpin effective and/ or superior performance in profession/occupational area." In Australia, competencybased curriculum models are now being used for the undergraduate training in a number of health professions including pharmacy, physiotherapy, dentistry and nursing (Gruppen, Mangrulkar, \& Kolars, 2012). Competency-based education is "a framework for designing and implementing education that focuses on the desired performance characteristics of health care professionals" (Gruppen et al., 2012, para. 1). Behavioural measures that "depend on integration of knowledge and skills derived from an aggregate of educational experiences and parts of the curriculum" (Cate \& Scheele, 2007) then become the focus of student assessments.

Table 1 depicts an adapted version of Miller's (1990) pyramid of competency development as might be applied to IPC. Theoretical microbiology and epidemiology form the knowledge base and IPC procedures such as hand hygiene and aseptic technique are the fundamental skills. 
Table 1

Stages of competency development (Liu, Curtis \& Crookes, 2014)

\begin{tabular}{|c|c|c|c|}
\hline Stage & Educational state & $\begin{array}{l}\text { Educational } \\
\text { goals/learner } \\
\text { outcomes }\end{array}$ & $\begin{array}{l}\text { Examples of educational } \\
\text { stage related to IPC }\end{array}$ \\
\hline 1 & Knows & Recitation of facts & $\begin{array}{l}\text { Describe the normal } \\
\text { microbial flora of the body }\end{array}$ \\
\hline 2 & Knows how & Applied knowledge & $\begin{array}{l}\text { Identify which type of } \\
\text { precaution category is } \\
\text { needed based on the route } \\
\text { of transmission of various } \\
\text { types of organisms }\end{array}$ \\
\hline 3 & Shows & Demonstration of skill & $\begin{array}{l}\text { Perform aseptic techniques } \\
\text { correctly }\end{array}$ \\
\hline \multirow[t]{2}{*}{4} & Does & $\begin{array}{l}\text { Performance/ } \\
\text { behaviour }\end{array}$ & $\begin{array}{l}\text { Apply policies, procedures } \\
\text { and guidelines relevant to } \\
\text { infection control when } \\
\text { presented with infection } \\
\text { control cases. }\end{array}$ \\
\hline & & & $\begin{array}{l}\text { Act as a role model to } \\
\text { HCWs, patients and } \\
\text { visitors by adhering to IPC } \\
\text { principles }\end{array}$ \\
\hline
\end{tabular}

Competence is recognised as "multi-dimensional and dynamic...[and] changes with time, experience and setting" (Frank et al., 2010, p. 641). It is our contention that student IPC competency development is influenced by multiple factors both internal and external to the curriculum and there is a need to avoid, or at least overcome, a piecemeal approach to curriculum design and implementation if IPC behaviour of graduates is to be optimised.

\section{Foundation knowledge - relevance and application}

O'Brien, Richards, Walton, Phillips, and Humphreys (2009) claimed that "appropriate knowledge is a starting point for improving practice and instilling the correct attitude to infection prevention" (p. 174). This view is supported by the comprehensive Centers for Disease Control and Prevention Guidelines ${ }^{1}$ which stated that HCWs need to understand the scientific rationale for the principles and practices of IPC in order to apply procedures correctly. An understanding of microbiology is fundamental foundational knowledge for IPC practice (Roark, 2005). In Australia, the foundational microbiology component of undergraduate

\footnotetext{
${ }^{1}$ http://wonder.cdc.gov/wonder/prevguid/prevguid.html
} 
nursing curricula is commonly situated in general bioscience subjects in the first year curriculum that also contain aspects of chemistry, anatomy and physiology (Davis, 2010). Nursing students' perceptions of science subjects being more difficult than other areas of the curriculum are well documented (Caon \& Treagust, 1993; Jordan, Davies, \& Green, 1999). Thornton (1997) postulated that students' integration and use of information is based on the perceived relevance of the content. Students tend to assess the relevance of science subjects in the curricula according to their interpretation of nursing, which for most, is a handson practical profession. Courtenay (1991) surveyed a group of third year nursing students $(n=140)$ and nurse teachers $(n=43)$ regarding their perceptions of subjects from the biological sciences. When asked to rank the subjects: anatomy, physiology, psychology, pharmacology, microbiology and sociology in order of importance in the preparation of a registered nurse, over half of the nursing students $(51 \%)$ considered anatomy to be the most important subject compared with $26 \%$ of their teachers. In contrast, just under half of the teachers $(41 \%)$ considered psychology the most important subject. Microbiology was ranked the least important subject area by both the teachers and the students. Students also considered microbiology and pharmacology the subjects that were hardest to learn.

The science education literature also identifies that, as with other areas of science, many nursing students find learning microbiology difficult (Courtenay, 1991). Becoming conversant with its specific terminology has been likened to learning a foreign language (Lumpkin Allen, 1997). A student's ability to learn and later apply microbiological and indeed scientific principles in general may be influenced by the perceived difficulty and relevance of the science subjects (Minasian-Batmanian, Lingard, \& Prosser, 2005). They may also be influenced by their preconceptions of science. Duit and Treagust (2003) noted:

...students do not come into science instruction without any preinstructional knowledge or beliefs about the phenomena and concepts to be taught. Rather, students already hold deeply rooted conceptions and ideas that are not in harmony with the science views or are even in stark contrast to them. (p. 671)

Alternative conceptions/misconceptions about scientific concepts can pose a further barrier to the learning of science (Committee on Undergraduate Science Education, 1997) and nurses' misconceptions of scientific principles such as pressure have been documented previously (Wilkes \& Batts, 1998). Further, it has been shown that HCWs' capacity to implement appropriate IPC precautions can be hampered by misconceptions about microbiological principles and inaccurate perceptions of risk (Gould, 1995; Prieto \& Clark, 2005). A positive correlation $(p<0.005)$ between knowledge of IPC precautions and understanding of theoretical microbiology was clearly demonstrated by Gould (1995) who used a combination of vignettes and a questionnaire to assess nurses' theoretical microbiology and infection control knowledge. Of concern, almost half of the participants $(47.85 \%)$ held misconceptions regarding the mode of transmission of the potentially lethal microorganism, methicillin-resistant Staphylococcus aureus, 
and subsequently would have applied inappropriate infection control precautions to safeguard themselves and other patients. Prieto and Clark (2005) explored the IPC views, priorities and practices of nurses and healthcare assistants $(n=18)$ in one English hospital. Although IPC policies were generally adhered to before and after patient care, there was consistent failure of participants to change gloves or wash hands during patient care, even if gloves had been in contact with a source of micro-organisms. The authors concluded that these findings could be explained, at least in part, by a lack of basic microbiology knowledge and inaccurate perceptions of risk. Similarly, statistical modelling undertaken by Whitby, McLaws and Ross (2006) found one significant predictor of hand washing behaviour to be belief in the benefit of the activity. The perceived importance of hand washing was based on nurses' assessment of the risk of infection:

Nurses believed that patients are a potential reservoir of infection because patients have little understanding of infection transmission. Nurses assessed the risk of infection due to contact with individual patients based on several criteria including the patient's diagnosis, physical appearance, and perceived general cleanliness. (p. 486)

The nexus between these factors is critical; misperceptions compounded by inaccurate risk perception can have implications for patient safety. For example, if a patient tests positive for a microorganism that is transmitted via droplets (airborne) but the HCW incorrectly believes the microorganism to be transmitted by direct (body fluid) contact, the $\mathrm{HCW}$ is likely to choose inappropriate personal protective equipment to wear. Alternatively, if the $\mathrm{HCW}$ makes assumptions that a microorganism is "not infectious" then they may be lax in their application of IPC measures. While assessment of student comprehension of theoretical microbiological concepts is likely to occur as part of the curriculum (commonly within the bioscience subjects), explicit identification of microbiological misconceptions seem to be far less common.

\section{Understanding behaviour - health behaviour theories}

Despite inclusion of microbiology education and infection control skill development in the early stages of undergraduate training, simply having an awareness of the need for good infection control is not enough to ensure translation into practice, nor is IPC practice necessarily related to increased levels of microbiology and/or infection control knowledge (Pittet, 2004). For example, even though $90.9 \%$ of nurses in Stein, Makarawo \& Ahmad's (2003) study considered washing hands before patient contact as "very important," only $58.7 \%$ reported "always" doing so. It is therefore reasonable to ask "why is this so?". Pittet (2004) claimed that behavioural motivations are an important cornerstone and key determinant of infection control alongside knowledge of microbiology and epidemiology. Therefore, IPC training must also be underpinned by these elements if changes in IPC performance are to be achieved. A recent study by Ward (2013) demonstrated the application of the Theory of Planned Behaviour (TPB) to nursing students' infection control intentions and behaviour (Figure 1) 


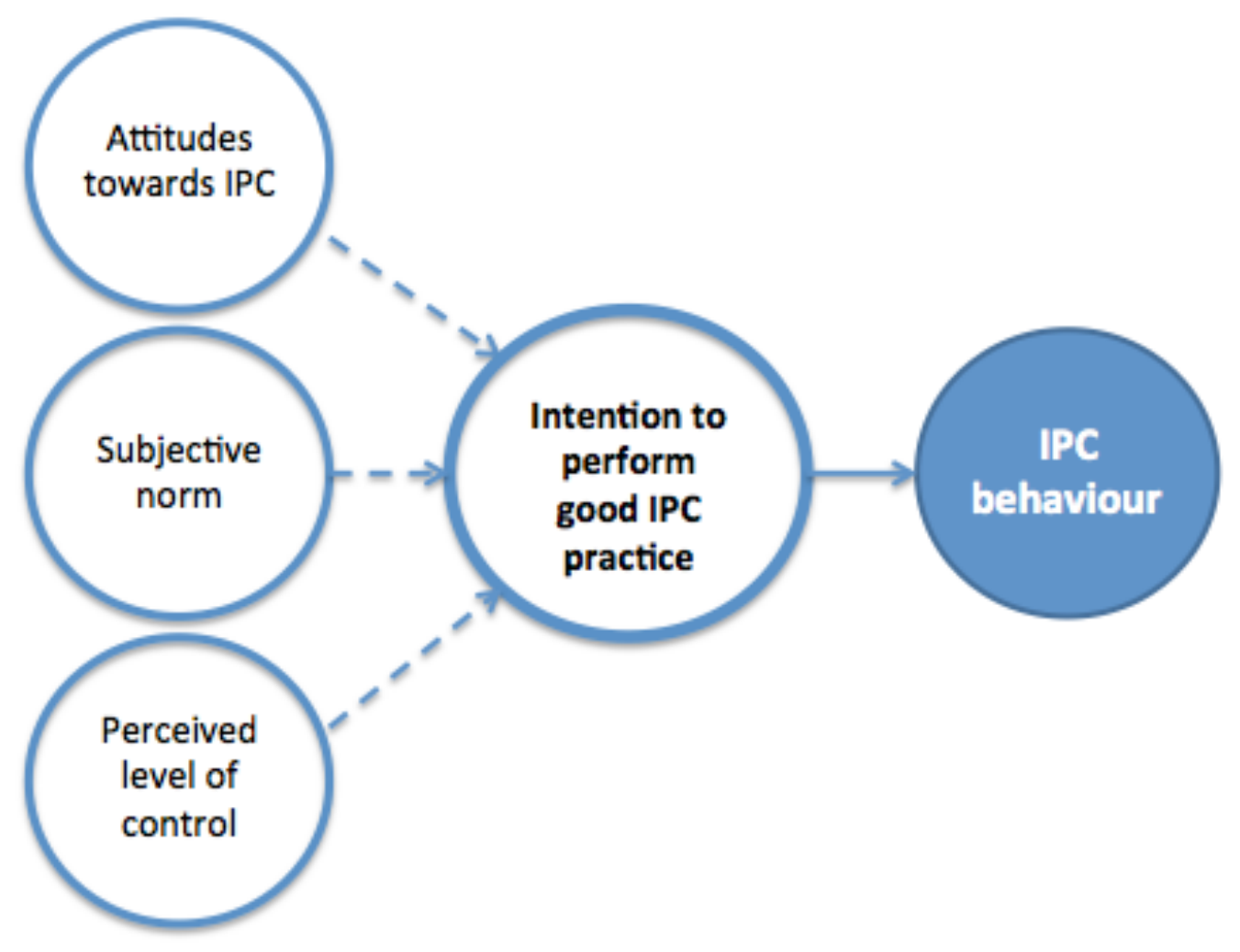

Figure 1. Application of Theory of Planned Behaviour to infection prevention and control practice (adapted from Ward, 2013)

According to Ward's (2013) social theory, Theory of Planned Behaviour (TPB), IPC behaviour can be directly linked to the intention to perform "good" IPC practice, which in turn, is influenced by attitudes about IPC, subjective norms (perceived pressure to perform IPC - strength of others' opinions) and perceived level of control. This study demonstrated that students' attitudes towards IPC prior to undertaking clinical placement can be positively influenced by microbiology/IC education, particularly when that education is structured in such as way as to highlight the risks and consequences of poor IPC practice. These positive intentions can, however, be negatively impacted by encountering a poor safety culture and/or role modelling of poor IPC practices by mentors, supervisors and colleagues in the workplace. Consequently, the perceived importance of IPC can be lessened and the necessity of such practices portrayed as "an additional extra rather than integral to care" (Ward, 2013, p. 304).

Another behavioural science theory, the Health Belief Model (HBM), has also been used as an explanatory framework to understand the infection prevention and control practices of health professionals (Brinsley, Sinkowitz-Cochran, Cardo, \& The CDC Campaign to Prevent Antimicrobial Resistance Team, 2005). According to the HBM (see Figure 2), behavioural change is influenced by a person's 
background (including socio-demographic factors), personal perceptions and cues to action.

\begin{tabular}{|c|c|c|}
\hline Background & $\begin{array}{l}\text { Sociodemographic Factors } \\
\text { - Age, Sex, Ethnicity } \\
\text { - Personality } \\
\text { - Knowledge }\end{array}$ & \\
\hline $\begin{array}{l}\text { Personal } \\
\text { perceptions }\end{array}$ & $\begin{array}{l}\text { Expectations } \\
\text { - } \quad \text { Perceived benefits } \\
\text { - } \quad \text { Perceived barriers } \\
\text { - } \quad \text { Perceived self-efficacy }\end{array}$ & $\begin{array}{c}\text { Threat } \\
\text { - } \quad \text { Perceived susceptibility } \\
\text { - } \quad \text { Perceived Severity }\end{array}$ \\
\hline Action & Cues to action & Behaviour change \\
\hline
\end{tabular}

Figure 2. The Health Belief Model (Brinsley et al., 2005, p. 176)

One of the factors predicting infection control practices, as identified by the $\mathrm{HBM}$, is perceived risk/susceptibility to infection and the perceived severity ("infectiousness" and consequences) of the microorganism and/or disease in question. If nurses don't perceive themselves to be at risk or perceive the microorganism to be of little threat to their own safety, they are less likely to adopt IPC precautions in that instance. Another predictive factor in this model of IPC behaviour is perceived benefits. Previous research has shown that as medical students progress towards the final years of their training they are less likely to see the value of hand hygiene for protecting themselves from acquiring antibioticresistant microorganisms and to prevent carrying microorganisms home (Limper et al., 2013).

The construct of self-efficacy (i.e. belief in one's ability to carry out a particular behaviour) is integral to a number of different behavioural models, including the HBM. Higher levels of self-efficacy facilitate increased effort and persistence towards particular behaviours (Kretzer \& Larson, 1998). Role modelling of good IPC practices and verbal reassurances from HCWs can enhance students' IPC self-efficacy. 


\section{Where to from here - potential strategies for enhancing graduates' IPC intentions, knowledge and practice}

When competent graduates are deployed, we expect that their work will contribute to certain outcomes. We expect that they will implement life-saving practices and demonstrate professional behaviours, perform those practices to standard, and make a public health impact by contributing to decreases in morbidity and mortality (Johnson, Fogarty, Fullerton, Bluestone, \& Drake, 2013).

Improving graduate readiness for the transition to infection control practice is likely to require a multi-faceted approach that addresses factors affecting IPC knowledge and intentions both inside and outside the classroom. If microbiology and infection control knowledge, perceptions of risk and perceived self-efficacy collectively underpin and inform IPC practice, then it follows that microbiology and behavioural science should collectively inform the future program planning and design, each component reinforcing the other in a far more integrated fashion.

For undergraduate nurses, the bioscience subjects (including microbiology) provide important foundational knowledge for the development of clinical judgement skills (Bednash, 2006). Given the well-documented struggle that nursing students have with these subjects, overcoming student perceptions of science as being difficult and irrelevant must be an important and ongoing consideration. The placement of theoretical microbiology content within bioscience subjects is nevertheless likely to potentiate the perceived irrelevance of that microbiology content. Benner, Sutphen, Leonard, and Day (2010) claimed that the extensive use of categorising frameworks in undergraduate nursing curricula whereby content is organised into distinct, decontexualised parts is "unnatural to the way that patients clinically present and how nurses think" (p. 5). Instead they advocated for the importance of incorporating contextualised examples such as open-ended case studies throughout the curriculum so that students "not only know the 'what' and 'how' of scientific knowledge, but 'when' to use it appropriately" (Handwerker, 2012, p. 6). The implementation of narratives and visual aids, based on common practice stories collected from nurse educators, has been reported as one strategy for enhancing the relevance of science in the clinical practice context (Dannenfeldt et al., 2009). Such contextualised learning can improve students' self-efficacy related to applying appropriate IPC in individual situations. Moreover, if compliance is related to perceived risk (as postulated by the HBM), it is important to ensure that students have accurate perceptions of risk informed by thorough knowledge of clinically relevant microbes. Thus, there is also a need to explicitly identify and address student misconceptions about microbiological principles.

An increase in microbiology knowledge alone, however, as previously established is unlikely to have a major impact on patient safety unless both practising and student nurses utilise this knowledge to implement appropriate IPC practices. Outside of the classroom, the strong influence of role models on student learning in the workplace during clinical placements and implementation of infection 
control practices when entering the profession has been noted by many other researchers (Cooper, 2001; Levett-Jones \& Lathlean, 2009). The development of an on-line pre-clinical placement learning plan developed collaboratively between students and their clinical supervisor can be helpful for establishing early communication and clarifying expectations of the clinical experience (Grace \& O'Neil, 2014). Both students and graduate nurses require a period of time to transition to their new role and develop a sense of professional identity. A busy workplace can make it difficult for students to develop the skills learnt as part of their pre-registration training and new graduates often report little opportunity to develop skills; rather, there is an expectation from the start for them to perform like more experienced nurses (Clark \& Holmes, 2007). High workload demands and time constraints can reduce students' sense of self-efficacy and perceived level of control which, according to Ward's (2013) Theory of Planned Behaviour (TPB) model will in turn negatively impact on their IPC intentions.

\section{Conclusion}

In summary, this paper establishes three areas of influence for graduates' IPC knowledge, intentions and practice: perceptions of science, health behaviour beliefs (perceived risk and self-efficacy) and applied knowledge (microbiology). New graduates in most health professions require an understanding of relevant microbiology but also an understanding of their own health beliefs, and a willingness to challenge these beliefs. The goal of re-thinking the microbiology/IC education of undergraduate health professionals is to enable accurate risk perception and maximise self-efficacy, thereby enhancing the development of competent, work-ready graduates who will contribute to lessening the burden of HAIs through improved IPC practice.

\section{References}

Allegranzi, B., \& Pittet, D. (2009). Role of hand hygiene in healthcare-associated infection prevention. Journal of Hospital Infection, 73(4), 305-315.

Bednash, G. (2006). Scientific underpinnings for professional nursing practice. Focus on Microbiology Education, 12(2), 5-6.

Brinsley, K. J., Sinkowitz-Cochran, R. L., Cardo, D. M., \& The C. D. C. Campaign to Prevent Antimicrobial Resistance Team. (2005). Assessing motivation for physicians to prevent antimicrobial resistance in hospitalized children using the Health Belief Model as a framework. American Journal of Infection Control, 33(3), 175-181. doi: 10.1016/j.ajic.2004.12.004

Burke, J. P. (2003). Infection control - A problem for patient safety. New England Journal of Medicine, 348(7), 651-656.

Caon, M., \& Treagust, D. (1993). Why do some nursing students find the science courses difficult? Journal of Nursing Education, 32(6), 255-259.

Cate, O. T., \& Scheele, F. (2007). Competency-based postgraduate training: can we bridge the gap between theory and clinical practice? Academic Medicine, 82, 542-547. 
Clark, T., \& Holmes, S. (2007). Fit for practice? An exploration of the development of newly qualified nurses using focus groups. International Journal of Nursing Studies, 44(7), 1210-1220. doi: 10.1016/j.ijnurstu.2006.05.010

Clements, A., Halton, K., Graves, N., Pettitt, A., Morton, A., Looke, D., \& Whitby, M. (2008). Overcrowding and understaffing in modern health-care systems: Key determinants in methicillin-resistant Staphylococcus aureus transmission. Lancet Infectious Diseases, 8(7), 427-434.

Collignon, P., Wilkinson, I., Gilbert, G., Grayson, M. L., \& Whitby, R. M. (2006). Health-care associated Staphylococcus aureus bloodstream infections: a clinical quality indicator for all hospitals. Medical Journal of Australia, 184, 404-406.

Committee on Undergraduate Science Education. (1997). Science teaching reconsidered: $A$ handbook. Washington, DC: National Academy Press.

Cooper, T. (2001). Educational theory into practice: development of an infection control link nurse programme. Nurse Education in Practice, 1(1), 35-41.

Courtenay, M. (1991). A study of the teaching and learning of the biological sciences in nurse education. Journal of Advanced Nursing, 16(9), 1110-1116. doi: 10.1111/j.1365-2648.1991.tb03372.x

Cox, J. L., Simpson, M. D., Letts, W., \& Cavanagh, H. M. A. (2014). Putting it into practice: Infection Control Professionals' perspectives on early career nursing graduates' microbiology and infection control knowledge and practice. Contemporary Nurse, 49(1-2).

Cruickshank, M., \& Ferguson, J. (Eds.) (2008). Reducing harm to patients from health care associated infection: The role of surveillance. Canberra, Australia: Australian Commission on Safety and Quality in Health Care. Retrieved from http://www.safetyandquality.gov.au/wp-content/uploads/2008/01/Reducing-Harm-toPatient-Role-of-Surveillance1.pdf

Dannenfeldt, G., Stewart, J., McHaffie, J., Gibson-van Marrewijk, K., Stewart, K., \& Hipkins, R. (2009). Addressing obstacles to success: implementing change in science delivery. Focus on Health Professional Education, 11(1), 41-48.

Davis, G. M. (2010). What is provided and what the registered nurse needs -- Bioscience learning through the pre-registration curriculum. Nurse Education Today, 30(8), 707712.

DeJoy, D. M., Gershon, R. R. M., \& Schaffer, B. S. (2004). Safety climate. Professional Safety, 49(7), 50-57.

Duit, R., \& Treagust, D. (2003). Conceptual change: A powerful framework for improving science teaching and learning. International Journal of Science Education, 25(6), 671-688.

Frank, J. R., Snell, L. S., Cate, O. T., Holmboe, E. S., Carraccio, C., Swing, S. R., . . . Harris, K. A. (2010). Competency-based medical education: theory to practice. Medical Teacher, 32(8), 638-645. doi: 10.3109/0142159X.2010.501190

Gammon, J., \& Gould, D. (2005). Universal precautions. A review of knowledge, compliance and strategies to improve practice. Journal of Research in Nursing, 10(5), 529-547.

Gould, D. (1995). Infection control: a survey to determine knowledge of nurses in a clinical setting. Nursing Standard, 9(36), 35-38. 
Gould, D. J., Drey, N. S., Moralejo, D., Grimshaw, J., \& Chudleigh, J. (2008). Interventions to improve hand hygiene compliance in patient care. Journal of Hospital Infection, 68(3), 193-202. doi: 10.1016/j.jhin.2007.11.013

Grace, S., \& O'Neil, R. (2014). Better prepared, better placement: An online resource for health students. Asia-Pacific Journal of Cooperative Education, 15(4), 291-304.

Gruppen, L., Mangrulkar, R., \& Kolars, J. (2012). The promise of competency-based education in the health professions for improving global health. Human Resources for Health, 10(43). doi:10.1186/1478-4491-10-43

Handwerker, S. (2012). Transforming nursing education: A review of current curricular practices in relation to Benner's latest work. International Journal of Nursing Education Scholarship, 9(1). doi: 10.1515/1548-923X.2510

Harbarth, S., Sax, H., \& Gastmeier, P. (2003). The preventable proportion of nosocomial infections: An overview of published reports. Journal of Hospital Infection, 54, 258266.

Huggonet, S., \& Pittet, D. (2000). Hand hygiene - beliefs or science? Clinical Microbiology and Infection, 6(7), 348-354.

Johnson, P., Fogarty, L., Fullerton, J., Bluestone, J., \& Drake, M. (2013). An integrative review and evidence-based conceptual model of the essential components of preservice education. Human Resources for Health, 11(42). doi:10.1186/1478-4491-1142

Jordan, S., Davies, S., \& Green, B. (1999). The biosciences in the pre-registration nursing curriculum: Staff and students' perceptions of difficulties and relevance. Nurse Education Today, 19(3), 215-226. doi: 10.1016/s0260-6917(99)80007-0

Kretzer, E. K., \& Larson, E. L. (1998). Behavioral interventions to improve infection control practices. American Journal of Infection Control, 26(3), 245-253. doi: 10.1016/S0196-6553(98)80008-4

Levett-Jones, T., \& Lathlean, J. (2009). 'Don't rock the boat': Nursing students' experiences of conformity and compliance. Nurse Education Today, 29(3), 342-349.

Limper, H. M., Barton, G., McGinty, M., Landon, E., O’Boyle, C., Reddy, S., \& Weber, S. G. (2013). Behavioral intention of physician trainees and medical students to practice hand hygiene. Infection Control and Hospital Epidemiology, 34(10), 11021105. doi: $10.1086 / 673146$

Liu, L.-M., Curtis, J., \& Crookes, P. A. (2014). Identifying essential infection control competencies for newly graduated nurses: A three-phase study in Australia and Taiwan. Journal of Hospital Infection, 86, 100-109.

Lumpkin Allen, D’M. A. (1997). The effects of computer-based multimedia lecture presentations on community college microbiology students' achievement, attitudes and retention. (9824854 Ph.D.), The University of Texas, Austin. Retrieved from http://dl.acm.org/author_page.cfm?id=81406597759\&coll=DL\&dl=ACM\&trk=0\&cfi $\mathrm{d}=626649554 \&$ cftoken $=45480630$

Miller, G. E. (1990). The assessment of clinical skills/competence/performance. Academic Medicine, 65, S63-S67.

Minasian-Batmanian, L. C., Lingard, J., \& Prosser, M. (2005). Differences in students' perceptions of learning compulsory foundation biochemistry in the health sciences 
professions. Advances in Health Science Education, 28(10), 279-290. doi: 10.1007/s10459-005-1404-7

Mutha, S., Jeffe, D. B., Kim, L. E., L’Ecuyer, P. B., Evanoff, B. A., \& Fraser, V. J. (1999). Healthcare workers' perceptions of occupational exposure. Infection Control and Hospital Epidemiology, 20(9), 592-593.

Nursing and Midwifery Board of Australia. (2006). National Competency Standards for the registered nurse. Retrieved from http://www.nursingmidwiferyboard.gov.au/Codes-Guidelines-Statements/CodesGuidelines.aspx

O'Brien, D., Richards, J., Walton, K. E., Phillips, M. G. A., \& Humphreys, H. (2009). Survey of teaching/learning of healthcare-associated infections in UK and Irish medical schools. Journal of Hospital Infection, 73(2), 171-175.

Pittet, D. (2004). The Lowbury lecture: Behaviour in infection control. Journal of Hospital Infection, 58(1), 1-13. doi: 10.1016/j.jhin.2004.06.002

Prieto, J., \& Clark, J. M. (2005). Contact precautions for Clostridium difficile and Methicillin-resistant Staphylococcus aureus (MRSA). Journal of Research in Nursing, 10(5), 511-526. doi: 10.1177/136140960501000508

Roark, J. (2005). Microbiology 101 for the ICP. Infection Control Today. Retrieved from http://www.infectioncontroltoday.com/articles/521 feat $1 . h t m l$

Scott, R. D. (2009). The direct medical costs of healthcare-associated infections in US hospitals and the benefits of prevention. Center for Disease Control and Prevention. Retrieved from http://www.cdc.gov/HAI/pdfs/hai/Scott_CostPaper.pdf

Sherwood, G. (2011). Integrating quality and safety science in nursing education and practice. Journal of Research in Nursing, 16(3), 226-240. doi: $10.1177 / 1744987111400960$

Stein, A. D., Makarawo, T. P., \& Ahmad, M. F. R. (2003). A survey of doctors' and nurses' knowledge, attitudes and compliance with infection control guidelines in Birmingham teaching hospitals. Journal of Hospital Infection, 54(1), 68-73.

Thornton, T. (1997). Attitudes towards the relevance of biological, behavioural and social sciences in nursing education. Journal of Advanced Nursing, 26(1), 180-186.

Ward, D. J. (2013). The application of the theory of planned behaviour to infection control research with nursing and midwifery students. Journal of Clinical Nursing, 22(1-2), 296-298. doi: 10.1111/j.1365-2702.2012.04327.x

Whitby, M., McLaws, M., \& Ross, M. (2006). Why healthcare workers don't wash their hands: A behavioural explanation. Infection Control and Hospital Epidemiology, 27(5), 484-492.

Wilkes, L., \& Batts, J. (1998). Nurses' understanding of physical science in nursing practice. Nurse Education Today, 18, 125-132. 